\title{
Investment Strategies for Flexible Resources
}

\author{
Jan A. Van Mieghem \\ J. L. Kellogg Graduate School of Management, Northwestern University, Evanston, Illinois 60208-2009
}

$\mathrm{T}_{\mathrm{r}}$

his article studies optimal investment in flexible manufacturing capacity as a function of product prices (margins), investment costs and multivariate demand uncertainty. We consider a two-product firm that has the option to invest in product-dedicated resources and / or in a flexible resource that can produce either product, but has to make its investment decision before demands are observed. The flexible resource provides the firm with a hedge against demand uncertainty, but at a higher investment cost than the dedicated resources. Our analysis highlights the important role of price (margin) and cost mix differentials, which, in addition to the correlation between product demands, significantly affect the investment decision and the value of flexibility. Contrary to the intuition also prevalent in the academic literature, we show that it can be advantageous to invest in flexible resources even with perfectly positively correlated product demands.

(Flexibility; Technology; Strategy; Capacity; Investment; Prices; Operational Hedging; Newsvendor Model)

\section{Introduction}

"The preserving of flexibility when faced with uncertainty" is no longer a neglected aspect of behavior under risk, as it was according to Jones and Ostroy (1984). Yet our understanding of flexibility still is based mainly on intuition that may be incomplete. Following the lead of Fine and Freund (1990), we study optimal investment in flexible manufacturing resources and consider a firm that faces uncertain demands for its two products. The firm has the option to invest in product-dedicated resources and in a flexible resource that is able to produce either product, but has to make its investment decision before demand is observed. The flexible resource provides the firm with a hedge against demand uncertainty but at the expense of a higher investment cost than the dedicated resources.

The results advanced in this article highlight the important role of price (or, more precisely, unit contribution margin) and cost mix differentials, which, in addition to the correlation between product demands, significantly affect the investment decision in flexible technology and the value of flexibility. The ability of product-flexible technology to deal with changes or uncertainty in demand mix (that is, relative proportions of

0025-1909/98/4408/1071\$05.00

Copyright (1) 1998, Institute for Operations Research

and the Management Sciences product quantities demanded) is well understood. This has led to a belief that flexible capacity provides no additional value when product demands are perfectly positively correlated. Fine and Freund [1990, p. 459] offer the argumentation behind this belief:

\footnotetext{
Because the demands for the two products move in lockstep, flexible capacity can only be useful if it can produce one product more cheaply than the dedicated capacity can. There will never be an opportunity to take advantage of the flexibility characteristic of the flexible capacity . . . when the two products' demands are perfectly positively correlated.
}

We will show that this belief is incomplete, if not false. Indeed, in addition to its adaptability to demand mix changes, product-flexible technology provides another opportunity for revenue improvement through its ability to exploit differentials in price (margin) mix. Product flexibility generates an option to produce and sell more of highly profitable products at the expense of less profitable products. More importantly, this option can remain valuable even with perfectly positively correlated product demand (i.e., when demand mix is constant and known with certainty).

This article is inspired and intimately related to the work by Fine and Freund (1990). Our research method, 
however, is different from their traditional discrete stochastic programming approach in that we use our "multi-dimensional newsvendor model," which was first presented in Harrison and Van Mieghem (1998). The resulting parsimonious descriptive model is amenable to analytic analysis and graphic interpretation, which allows our results to be easily taught and remembered. Its multi-dimensionality enriches the traditional newsvendor model by incorporating product, resource and demand differentiation through price and cost vectors, a technology matrix and a multivariate demand distribution. The intent of this article is to build new theory and intuition on the benefits of product flexibility to hedge against demand uncertainty by highlighting the role of price and cost mix differentials in addition to demand correlation. For our purposes here it suffices to analyze a one-period model. As such, our approach may be too highly stylized to serve as a practical decision support system, which may need to consider more complex models for which one must resort to numerical methods, cf. Caulkins and Fine (1990), Li and Tirupati (1994). Alternately, multi-period extensions may be analyzed using our recent theory on quasi-reversible multi-dimensional investment under uncertainty (Eberly and Van Mieghem 1997, Harrison and Van Mieghem 1998). (Please refer to Fine and Freund (1990) and Van Mieghem (1997) for general definitions of "flexibility" and a more extensive literature review.)

This article is organized as follows. The next section presents the model and $\S 3$ discusses the optimal investment position using our multidimensional newsvendor solution. Section 4 shows that the optimal investment strategy has one of three distinct forms and analyzes the sensitivity of the optimal investment to changes in prices (margins) and investment costs. Finally, §5 examines how the optimal investment depends on the multivariate demand distribution and presents closed-form solutions for perfectly correlated product demand, emphasizing the role of price differentials. (All proofs can be found in Van Mieghem (1997).)

We conclude this introduction with some notational conventions. We will not distinguish in notation between scalars and vectors. All vectors are assumed to be column vectors, and primes denote transposes. Vector inequalities should be interpreted componentwise.
As usual, $E$ and $\nabla$ denote the expectation and gradientvector operators.

\section{Modeling Investment in Flexible Resources}

Consider a firm that has the option to invest in two product-dedicated resources and one flexible resourcerespectively labeled resources 1,2 and 3-to manufacture two products. First, the firm must decide on a nonnegative vector of resource capacity levels $K \in \mathbb{R}_{+}^{3}$ for production, before the product demand vector $D \in \mathbb{R}_{+}^{2}$ is observed. After demand is observed, the firm chooses, constrained by its earlier resource investment, a vector $x=\left(y_{1}, y_{2}, z_{1}, z_{2}\right) \in \mathbb{R}_{+}^{4}$ of production quantities, where $y_{j}+z_{j}$ is the total production quantity of product $j$ and $y_{j}$ and $z_{j}$ represent the quantities produced on the product- $j$-dedicated and flexible resource respectively. This multi-stage decision problem, also known as a stochastic program with recourse, is characteristic of real option models: first invest in capabilities, then receive some additional information, and finally exploit capabilities optimally contingent on the revealed information.

The firm's manufacturing process and production decisions are modeled as follows. Having chosen a capacity vector $K$ and observed a demand vector $D$, the firm chooses its production vector $x$ as the optimal solution of the following product mix problem so as to maximize operating profit:

$$
\begin{gathered}
\max _{y, z \in \mathbb{R}_{+}^{2}} p_{1}\left(y_{1}+z_{1}\right)+p_{2}\left(y_{2}+z_{2}\right) \\
\text { subject to } y_{1} \leq K_{1}, \\
y_{2} \leq K_{2}, \\
z_{1}+z_{2} \leq K_{3}, \\
y_{1}+z_{1} \leq D_{1}, \\
y_{2}+z_{2} \leq D_{2},
\end{gathered}
$$

where $p \in \mathbb{R}_{+}^{2}$ is a price or margin vector whose $j$ th component represents the unit contribution margin for product $j$ (that is, sales price minus variable cost of production). The optimal objective value of the product mix problem (1) through (6) is the maximal operating profit and is denoted by $\pi(K, D)=\left(p_{1}, p_{2}, p_{1}, p_{2}\right)^{\prime} x(K, D)$, 
where $x(K, D)$ is an associated optimal production vector. To keep the number of parameters manageable, we have implicitly made two assumptions in the product mix problem. First, product $j$ variable production costs on the associated dedicated resource and on the flexible resource are identical. Second, because the contribution margins do not depend on the production quantities chosen, the firm is assumed to be a price taker in both the output and factor markets. Notice that although (4) seems to indicate that both products require an equal amount of the flexible resource to produce one unit, this is without any loss of generality since in this model it is just a matter of how units are defined.

Assuming that the firm starts with no initial resources, it incurs an investment cost $C(K)$ if it chooses a capacity vector $K$. For simplicity we assume that investment costs are linear,

$$
C(K)=c^{\prime} K,
$$

where $c \in \mathbb{R}_{+}^{3}$ is a vector of marginal investment costs, but the results presented below directly generalize to any convex function $C$. In order for the model to be realistic and to yield interesting results, we assume that flexible capacity is more expensive than dedicated, yet sufficiently inexpensive to be a viable alternative: $0<c_{1}, c_{2}$ $<c_{3}<c_{1}+c_{2}$. Also, it should be economically justified to produce both products, i.e., $c_{1}<p_{1}$ and $c_{2}<p_{2}$, where the more profitable product is given label 1 , so that $p_{1}$ $\geq p_{2}>0$, and the price (or margin) differential will be denoted by $\Delta p=p_{1}-p_{2} \geq 0$. Finally, demand uncertainty is represented by a probability measure $P$ over the demand space $\mathbb{R}_{+}^{2}$. For simplicity we assume that $D$ is a continuous random vector that is finite with probability one and that has a joint probability density function $g$, which is positive over its support. The firm seeks a strategy of investment and production that maximizes

$$
V(K)=E \pi(K, D)-C(K),
$$

the expected value of operating profits minus resource investment costs. We denote the maximal value of $V(\cdot)$ by $V^{*}$ and call any maximizer of $V(\cdot)$ an optimal investment vector.

It is straightforward to incorporate demand shortage penalties and capacity salvage values into the model as follows. Assume product $j$ carries a shortage penalty $\operatorname{cost} c_{P, j} \geq 0$ for each unit of demand that is not satisfied (i.e., for each unit of $D_{j}>y_{j}+z_{j}$ ) and a unit of resource $i$ has a salvage value $c_{S, i}<c_{i}$ at the end of the period. Then all results presented in this article remain valid if we inflate unit contribution margins $p$ to $p+c_{P}$, deflate marginal investment costs $c$ to $c-c_{S}$ and decrease the operating profit $\pi(K, D)$ by $c_{P}^{\prime} D$ (which also decreases $V(K)$ by the constant $\left.E c_{P}^{\prime} D\right)$.

\section{The Multidimensional Newsvendor Solution}

Like most multistage decision problems, our model is analyzed backward by first solving for the optimal contingent production decisions $x(K, D)$, and the associated three-vector $\lambda(K, D)$ of optimal dual variables, or shadow prices, of the capacity constraints (2) through (4) in the product mix problem. Parametric analysis of that linear program (i.e., using the Simplex method) leads us to partition the demand space $\mathbb{R}_{+}^{2}$ given a capacity vector $K \in \mathbb{R}_{+}^{3}$ into five domains as shown in Figure 1, where the thick-lined $\Omega_{0}(K)$ is the firm's production capacity region. This allows us to express the optimal contingent primal and dual variables: $x=\left(D_{1}, K_{2}, 0, K_{3}\right)^{\prime}$ and $\lambda$ $=\left(0, p_{2}, p_{2}\right)^{\prime}$ if $D \in \Omega_{1}(K), x=\left(K_{1}, K_{2}, D_{1}-K_{1}, K_{3}\right.$ $\left.-D_{1}+K_{1}\right)^{\prime}$ and $\lambda=\left(p_{2}, p_{2}, p_{2}\right)^{\prime}$ if $D \in \Omega_{2}(K), x=\left(K_{1}\right.$, $\left.K_{2}, K_{3}, 0\right)^{\prime}$ and $\lambda=\left(p_{1}, p_{2}, p_{1}\right)^{\prime}$ if $D \in \Omega_{3}(K), x=\left(K_{1}\right.$, $\left.D_{2}, K_{3}, 0\right)^{\prime}$ and $\lambda=\left(p_{1}, 0, p_{1}\right)^{\prime}$ if $D \in \Omega_{4}(K)$; and $\lambda=0$ and any vector $x$ of production quantities satisfying $y_{1}$ $+z_{1}=D_{1}$ and $y_{2}+z_{2}=D_{2}$ is optimal if $D \in \Omega_{0}(K)$.

From basic linear programming theory we know that $\pi(\cdot, \cdot)$, and thus $V(\cdot)$, is concave so that the KuhnTucker first-order conditions are necessary and sufficient to maximize $V(\cdot)$. In Harrison and Van Mieghem (1998), we show that differentiation and expectation can be interchanged so that $\nabla E \pi(\cdot, D)=E \lambda(\cdot, D)$. Finally, because the shadow price vector is constant in each domain $\Omega_{i}(K)$, we can express the optimality equations in terms of the dual variables as follows.

Proposition 1. An investment vector $K^{*} \in \mathbb{R}_{+}^{3}$ is optimal if and only if there exists a $\nu \in \mathbb{R}_{+}^{3}$ such that

$$
\begin{aligned}
& \left(\begin{array}{c}
0 \\
p_{2} \\
p_{2}
\end{array}\right) P\left(\Omega_{1}\left(K^{*}\right)\right)+\left(\begin{array}{c}
p_{2} \\
p_{2} \\
p_{2}
\end{array}\right) P\left(\Omega_{2}\left(K^{*}\right)\right) \\
& +\left(\begin{array}{c}
p_{1} \\
p_{2} \\
p_{1}
\end{array}\right) P\left(\Omega_{3}\left(K^{*}\right)\right)+\left(\begin{array}{c}
p_{1} \\
0 \\
p_{1}
\end{array}\right) P\left(\Omega_{4}\left(K^{*}\right)\right)=c-\nu,
\end{aligned}
$$




$$
\nu^{\prime} K^{*}=0
$$

It is readily shown that the optimal investment level $K^{*}$ is unique. Proposition 1 greatly enhances the intuitive content of the model by providing a solution technique with a graphical interpretation. The optimal investment is found by superimposing the multivariate demand distribution onto Figure 1 and adjusting the thick lines of the feasible region (these are determined by $K$ ) such that the probabilities of the four domains $\Omega_{1}$, $\ldots, \Omega_{4}$ offset the marginal investment cost $c$ as in the optimality Equation (9). Generalizing the language of the familiar one-dimensional newsvendor model, one can say that it is optimal to invest up to a critical "fractile" of the multivariate demand distribution, thereby balancing "overage costs" with "underage costs."

\section{How Optimal Investment Depends on Costs and Prices}

Proposition 2 will highlight the role of investment costs by showing that the optimal investment strategy must take one of the following forms: (i) invest in dedicated resources only, (ii) do not invest in dedicated capacity for product 2 but invest in dedicated capacity for product 1 as well as in flexible capacity, or (iii) invest in all three types of capacity. No other combinations of investment can be optimal. Before we

Figure 1 The Total Production Quantities (Product Sales) $q=\left(y_{1}+z_{1}\right.$, $\left.y_{2}+z_{2}\right)$ and Shadow Prices $\lambda$ Depend on the Capacity $K$ and the Demand $D$

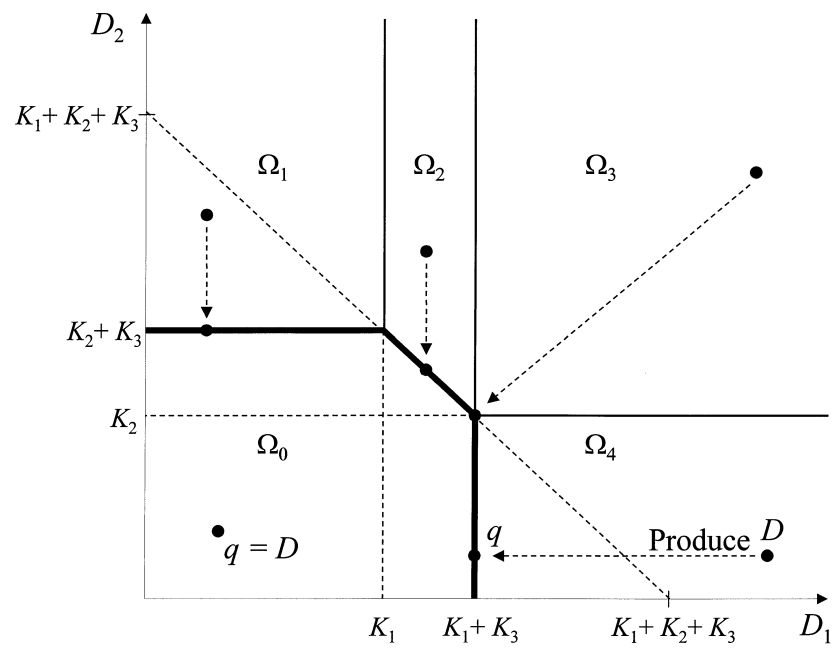

can explicitly write out the firm's optimal investment policy in terms of the model primitives, we must first define two non-negative threshold values $\underline{c}_{3}$ and $\overline{c_{3}}$, as follows. Strategy (i) corresponds to a boundary solution of Proposition 1 of the form $\bar{K}=\left(\bar{K}_{1}, \bar{K}_{2}, 0\right)$ that is the unique solution to

$$
\begin{aligned}
& p_{1} P\left(\Omega_{3}(\bar{K})\right)+p_{1} P\left(\Omega_{4}(\bar{K})\right)=p_{1} P\left(D_{1}>\bar{K}_{1}\right)=c_{1}, \\
& p_{2} P\left(\Omega_{1}(\bar{K})\right)+p_{2} P\left(\Omega_{3}(\bar{K})\right)=p_{2} P\left(D_{2}>\bar{K}_{2}\right)=c_{2},
\end{aligned}
$$

while the third optimality equation reduces to $c_{3}>\overline{c_{3}}$ where

$$
\bar{c}_{3}=c_{1}+c_{2}-p_{2} P\left(\Omega_{3}(\bar{K})\right) .
$$

Strategy (ii) is a boundary solution of the form $\underline{K}=\left(\underline{K}_{1}\right.$, $0, \underline{K}_{3}$ ) which is the unique solution to

$$
\begin{gathered}
p_{2} P\left(\Omega_{2}(\underline{K})\right)+p_{1} P\left(\Omega_{3}(\underline{K})\right)=c_{1}, \\
p_{2} P\left(\Omega_{1}(\underline{K})\right)+p_{2} P\left(\Omega_{2}(\underline{K})\right)+p_{1} P\left(\Omega_{3}(\underline{K})\right)=c_{3},
\end{gathered}
$$

while the second optimality equation reduces to $c_{3}<\overline{c_{3}}$ where

$$
\underline{c}_{3}=c_{2}+P\left(\Omega_{3}(\underline{K})\right) \Delta p .
$$

Conditions (12) and (14) yield that $0 \leq p_{2} P\left(\Omega_{3}(\bar{K})\right)$ $\leq c_{2}$ and $p_{1} P\left(\Omega_{3}(\underline{K})\right) \leq c_{1}$, respectively. Because $\Delta p$ $=p_{1}-p_{2} \geq 0$, it follows that $c_{1} \leq \overline{c_{3}} \leq c_{1}+c_{2}$ and $c_{2}$ $\leq \underline{c}_{3} \leq \bar{c}_{3}$. We can now state the main result in Proposition 2 .

PROPOSITION 2. The optimal investment strategy has one of three distinct forms, depending on the marginal cost of flexibility $c_{3}$ :

(i) If $c_{3}>\overline{c_{3}}$, it is optimal to invest only in dedicated resources and $K^{*}=\bar{K}$.

(ii) If $c_{3}<\underline{c}_{3}$, it is optimal to invest only in the product-1 dedicated resource and the flexible resource and $K^{*}=\underline{K}$. This requires a positive price differential $\Delta p>0$.

(iii) Otherwise, it is optimal to invest in all three resources and $K^{*}$ solves (9) with $\nu=0$.

Because $\max \left(c_{1}, c_{2}\right) \leq \overline{c_{3}} \leq c_{1}+c_{3}$, there are values for $c_{3}$ that make strategy (i) and/or (iii) optimal (both are possible if $\left.\max \left(c_{1}, c_{2}\right)<\overline{c_{3}}<c_{1}+c_{3}\right)$. A positive price differential $\Delta p>0$, however, is necessary, but not sufficient, for strategy (ii) to be optimal: because we cannot guarantee that $\underline{c}_{3} \geq c_{1}$, the problem parameters $p, c_{1}, c_{2}$ and $P$ may be such that strategy (ii) is never optimal for 
any value of $c_{3}$. Besides understanding the pivotal role of the marginal cost of the flexible resource, it is also interesting to see how the optimal investment level changes as the entire marginal cost vector $c$ changes.

Proposition 3. The optimal value $V^{*}$ is a nonincreasing convex function of the marginal capacity costs $c$ with gradient $\nabla_{c} V^{*}=-K^{*} \leq 0$, and the optimal investment vector $K^{*}$ has cost sensitivity terms

$$
\nabla_{c} K^{* \prime}=\left(\begin{array}{ccc}
-\left(\alpha_{1}+\alpha_{2}+\alpha_{4}\right) & -\alpha_{1} & \alpha_{1}+\alpha_{2} \\
-\alpha_{1} & -\left(\alpha_{1}+\alpha_{3}+\alpha_{5}\right) & \alpha_{1}+\alpha_{3} \\
\alpha_{1}+\alpha_{2} & \alpha_{1}+\alpha_{3} & -\left(\alpha_{1}+\alpha_{2}+\alpha_{3}+\alpha_{6}\right)
\end{array}\right),
$$

where $\alpha\left(K^{*}\right) \in \mathbb{R}_{+}^{6}$ and $\alpha>0$ under optimal strategy (iii); $\alpha_{2}, \alpha_{4}, \alpha_{6}>0$ and all other $\alpha_{j}=0$ under strategy (ii); and $\alpha_{4}, \alpha_{5}>0$ and all other $\alpha_{j}=0$ under strategy (i).

Proposition 3 extends Lemma 2 and Theorem 2 of Fine and Freund (1990) to our multidimensional newsvendor solution with continuous demand distribution and has a similar interpretation. It is not surprising that the optimal value $V^{*}$ and the optimal investment level $K_{j}^{*}$ of resource $j$ do not increase as the marginal cost of resource $j$ increases. The more interesting result is the substitution effect implicit in the off-diagonal terms of (17): as the marginal cost of a dedicated resource increases, the decrease in the optimal investment level of both dedicated resources is partially offset by an increase in the optimal level of the flexible resource, and vice versa for an increase in the marginal cost of the flexible resource. Summing terms, however, shows that the substitution is incomplete:

$$
\begin{aligned}
& \frac{\partial}{\partial c_{1}}\left(K_{1}^{*}+K_{2}^{*}+K_{3}^{*}\right) \\
& \quad \leq \frac{\partial}{\partial c_{1}}\left(K_{1}^{*}+K_{3}^{*}\right) \leq 0 \leq \frac{\partial}{\partial c_{1}}\left(K_{2}^{*}+K_{3}^{*}\right),
\end{aligned}
$$

and similar relations hold for $c_{2}$ and $c_{3}$. The impact of price (margin) changes is expressed in Proposition 4.

Proposition 4. The optimal value $V^{*}$ is an increasing convex function of the price (margin) vector $p$ with gradient $\nabla_{P_{i}} V^{*}=E\left(y_{i}+z_{i}\right)>0$, and the optimal investment vector $K^{*}$ has price sensitivity terms

$$
\nabla_{p} K^{* \prime}=\left(\begin{array}{ccc}
\beta_{2} & -\beta_{1} & \beta_{1}+\beta_{3} \\
-\beta_{5}+\beta_{6}+\beta_{7} & \beta_{4} & \beta_{5}-\beta_{6}+\beta_{8}-\beta_{9}
\end{array}\right),
$$

where $\beta\left(K^{*}\right) \in \mathbb{R}_{+}^{9}$ and $\beta>0$ with $\beta_{4}+\beta_{7}+\beta_{8}>\beta_{9}$ under optimal strategy (iii); $\beta_{2}, \beta_{3}, \beta_{5}, \beta_{7}, \beta_{8}>0$ and all other $\beta_{j}=0$ under strategy (ii); and $\beta_{2}, \beta_{4}>0$ and all other $\beta_{j}=0$ under strategy (i).

Obviously, a higher price (margin) $p_{j}$, is beneficial and warrants a higher investment level $K_{j}^{*}$ in the corresponding dedicated resource. More interesting is the impact of an increase in $p_{1}$, or equivalently in the price differential $\left.\Delta p\right|_{p_{2}=c t e}$, on the flexibility investment. Such increase asks for a substitution of dedicated product 2 (the less profitable product) capacity into flexible capacity with a positive net effect:

$$
\left.\frac{\partial}{\partial \Delta p}\left(K_{2}^{*}+K_{3}^{*}\right)\right|_{p_{2}=c t e}=\beta_{3}>0 .
$$

Vice-versa for an increase in $p_{2}$ under strategy (ii), though the effect is less-pronounced under strategy (i), but total capacity is always increasing:

$$
\frac{\partial}{\partial p_{i}}\left(K_{1}^{*}+K_{2}^{*}+K_{3}^{*}\right)>0 .
$$

The impact of this substitution on price-mix exploitation will be explained in the next section.

\section{How Optimal Investment Depends on Demand Uncertainty}

The optimal investment level $K^{*}$ and the threshold costs $\underline{c}_{3}$ and $\overline{c_{3}}$ depend not only on the contribution margins $p$ and the marginal investment $\operatorname{costs} c$, but also on the entire demand distribution. Some insights follow directly from the graphical interpretation of the solution: a translation of the demand distribution by $\left(\delta_{1}, \delta_{2}\right)$ is absorbed by a corresponding shift in the optimal investment level of the dedicated resources without affecting the optimal level of the flexible resource: $K^{*}$ changes to $\left(K_{1}^{*}+\delta_{1}, K_{2}^{*}+\delta_{2}, K_{3}^{*}\right)$. Also, as the level of uncertainty in the demand distribution decreases, the optimal level of the dedicated resources tends to the mean demand while the optimal level of the flexible resource tends to 
The Optimal Investment Strategies When Demands Are Perfectly Positively Correlated, $\Delta p>0$ and $c_{1} / p_{1}<c_{2} / p_{2}$ for Three Scenarios: High Cost of Flexibility (Left), Medium Cost (Center), Low Cost (Right)

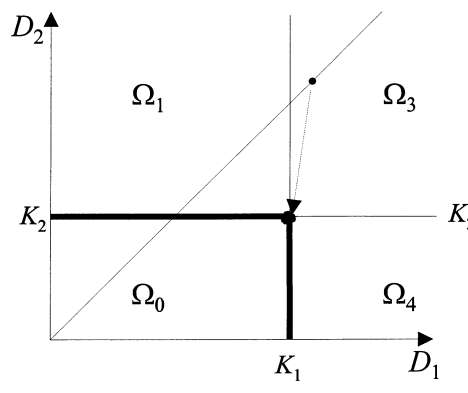

zero. Indeed, the flexible resource provides us with a real option that can be exercised after uncertainty is realized. If there is no uncertainty this option has no value and one will invest in dedicated resources only: $K^{*}$ $=\left(D_{1}, D_{2}, 0\right)$. Dedicated resources seem to serve as "base capacity," whereas the flexible resource serves as an optimal cost/benefit response to variability in demand. Because the optimal investment solution depends on the entire shape of the demand distributiona familiar result of the newsvendor model-it cannot be expressed in terms of a few demand parameters, such as the mean demand or its variance, only. Thus it is difficult to draw any general conclusions on the impact of the demand distribution on the optimal investment strategy. The following sensitivity result, however, may help to illustrate how the optimal investment level depends on a particular parameter $\theta$ of the demand distribution.

Proposition 5. Consider a parametric family of demand density functions $g(\cdot \mid \theta)$, assuming $g(\cdot \mid \theta)$ is differentiable w.r.t. the scalar parameter $\theta$. Let the domains $\Omega_{i}=\Omega_{i}\left(K^{*}\right)$ for $i=1$, $\ldots, 4$ and $\alpha\left(K^{*}\right)$ be defined as above and define the vector J by

$$
J_{i}=\int_{\Omega_{i}} \frac{\partial}{\partial \theta} g(z \mid \theta) d z .
$$

Then the $\theta$-sensitivity of the optimal investment level $K^{*}$ under strategy (iii) is

$$
\begin{aligned}
& \frac{\partial}{\partial \theta} K^{*} \\
& \quad=\left(\begin{array}{cccc}
-\alpha_{2} p_{2} & \left(\alpha_{1}+\alpha_{4}\right) p_{2} & \alpha_{4} p_{1}+\alpha_{1} p_{2} & \alpha_{4} p_{1} \\
\alpha_{5} p_{2} & \left(\alpha_{1}+\alpha_{5}\right) p_{2} & \left(\alpha_{1}+\alpha_{5}\right) p_{2}-\alpha_{3} \Delta p & -\alpha_{3} p_{1} \\
\left(\alpha_{2}+\alpha_{6}\right) p_{2} & \left(\alpha_{6}-\alpha_{1}\right) p_{2} & \alpha_{6} p_{1}+\alpha_{3} \Delta p-\alpha_{1} p_{2} & \left(\alpha_{3}+\alpha_{6}\right) p_{1}
\end{array}\right) J .
\end{aligned}
$$

Comparing the signs in (19) shows that also here a substitution effect is present between dedicated and flexible capacity. A general study of the impact of demand parameter $\theta$ requires an analysis of the sum of products of the parameters $J$ and $\alpha$, each of which itself is a complex function of the demand distribution. For the remainder of this article, we will focus on the correlation between the two product demands.

Proposition 6. Let product demands be perfectly positively correlated: $P\left(\left\{D_{1}=D_{2}\right\}\right)=1$.

(a) If $\Delta p=0$, or if $\Delta p>0$ with $c_{1} / p_{1} \geq c_{2} / p_{2}$, then $\overline{c_{3}}$ $=\max \left(c_{1}, c_{2}\right)$ and case (i) of Proposition 2 occurs: it is optimal to invest only in dedicated resources regardless of the cost of flexible capacity $c_{3}$ (and of a positive price differential $\Delta p)$.

(b) If $\Delta p>0$ with $c_{1} / p_{1}<c_{2} / p_{2}$, then

$$
\max \left(c_{1}, c_{2}\right)<\overline{c_{3}}=\frac{\Delta p}{p_{1}} c_{1}+c_{2}<c_{1}+c_{2}
$$

and cases (i) and (iii) of Proposition 2 can occur (case (ii) may never occur), always with $K_{1}^{*}>K_{2}^{*}+K_{3}^{*}$. The optimality equations for $K^{*}$ in case (iii) simplify to

$$
\begin{gathered}
P\left(K_{1}^{*}<D_{1}\right)=\frac{c_{1}+c_{2}-c_{3}}{p_{2}}, \\
P\left(K_{1}^{*}+K_{3}^{*}<D_{1}\right)=\frac{c_{3}-c_{2}}{\Delta p} \text { and } \\
P\left(K_{2}^{*}+K_{3}^{*}<D_{1}\right)=\frac{c_{2}}{p_{2}} .
\end{gathered}
$$

Proposition 6 emphasizes the significance of the "price" differential $\Delta p$ in the flexibility decision. Contrary to intuition, it shows that it is optimal to invest in 
flexible capacity if there is a positive price (margin) differential $\Delta p>0$ and $c_{1} / p_{1}<c_{2} / p_{2}$ and if $c_{3}<\overline{c_{3}}$. Figure 2 provides a graphical explanation of how flexibility can yield superior performance in that case, even with perfectly positively correlated demands. It portrays the shape of the feasible regions created by optimal investment strategies under three different parameter combinations that differ only in the cost of flexible capacity. Only demand pairs on the 45 degree line are possible. To compare the three scenarios, consider the specific demand outcome and the corresponding optimal production decision that are connected by the arrow in the pictures. In scenario 1 at the left, the cost of flexible capacity is high and it is optimal to invest only in dedicated capacity, yielding a rectangular feasible region. If the cost of flexible capacity decreases below $\overline{c_{3}}$, we arrive at scenario 2 in the center. Here, investing in the flexible resource creates the option to produce more (compared to the investment in dedicated resources only shown by the dotted rectangular feasible region) of the more profitable product 1 at the expense of the less profitable product 2 when demand falls in domain $\Omega_{2}$. The associated profit gain outweighs the increased investment costs so that it is optimal to invest in the flexible resource even though the product demands move in lockstep. As we move from scenario 1 to scenario 2, the maximal product 1 capacity increases, while the maximal product 2 capacity remains constant $\left(K_{2}\right.$ in scenario 1 equals $K_{2}+K_{3}$ in scenario 2 ). This is in agreement with Proposition 3: as $c_{3}$ decreases, the increase in $K_{3}^{*}$ partially substitutes the decrease in dedicated capacity. Finally, if the cost of flexibility decreases so much that $K_{2}$ becomes zero in scenario 2 (while $P\left(\Omega_{3}\right)>0$ ), scenario 3 at the right applies ${ }^{1}$.

Notice that the simple necessary and sufficient condition for a firm to invest in flexible resources is independent of the particular probability distribution of $D_{1}$ $=D_{2}$. It thus is independent of the level of variability or "risk" in demand, as long as some variability is present. Obviously, as said earlier, the presence of uncertainty remains key.

\footnotetext{
${ }^{1}$ While scenarios 1 and 2 always occur, scenario 3 may never occur, namely if $K_{2}$ remains strictly positive in scenario 2 when the cost of flexibility is at its minimum $\left(=\max \left(c_{1}, c_{2}\right)\right)$.
}

PROPOSITION 7. Let product demands be perfectly negatively correlated: $P\left(\left\{D_{1}+D_{2}=k>0\right\}\right)=1$, and let

$$
\overline{c_{3}}=p_{2}+\frac{\Delta p}{p_{1}} c_{1} \quad \text { and } \quad c_{3}^{*}=p_{1}-\frac{\Delta p}{p_{2}} c_{2} .
$$

(a) If $c_{1} / p_{1}+c_{2} / p_{2}>1$ and $c_{3}>\bar{c}_{3}$, then the optimal strategy invests in dedicated resources only: case (i) of Proposition 2 occurs with $K_{1}^{*}+K_{2}^{*}<k$.

(b) If $c_{1} / p_{1}+c_{2} / p_{2}>1$ and $\overline{c_{3}} \geq c_{3}>c_{3}^{*}$ (this requires a positive price differential $\Delta p>0$ ), then the optimal strategy invests in all three resources with $K_{1}^{*}+K_{2}^{*}+K_{3}^{*}<k$ and

$$
\begin{gathered}
P\left(D_{1}<K_{1}^{*}\right)=\frac{c_{3}-c_{1}}{p_{2}}, \\
P\left(D_{1}<K_{1}^{*}+K_{3}^{*}\right)=\frac{p_{1}-c_{3}}{\Delta p} \text { and } \\
P\left(D_{1}<k-K_{2}^{*}\right)=\frac{c_{2}}{p_{2}} .
\end{gathered}
$$

(c) If $c_{1} / p_{1}+c_{2} / p_{2}>1$ and $c_{3}^{*} \geq c_{3}$, or if $c_{1} / p_{1}+c_{2} /$ $p_{2} \leq 1$, then the optimal strategy invests in all three resources with $K_{1}^{*}+K_{2}^{*}+K_{3}^{*}=k$ and

$$
\begin{gathered}
P\left(D_{1}<K_{1}^{*}\right)=\frac{c_{3}-c_{1}}{p_{2}} \text { and } \\
P\left(K_{1}^{*}+K_{3}^{*}<D_{1}\right)=\frac{c_{3}-c_{2}}{p_{1}} .
\end{gathered}
$$

Figure 3 portrays the shape of the feasible regions created by the optimal investment strategies under the three different possible scenarios of Proposition 7: high cost of flexible capacity (left picture), medium cost (middle), and low cost (right). Now, only demand pairs on the $-45^{\circ}$ line are possible. Although more in line with intuition, Proposition 7 shows that if both products are sufficiently profitable (as measured by $c_{1} / p_{1}+c_{2} / p_{2}$ $\leq 1$ ), it is optimal to invest in the flexible resource regardless of its marginal investment cost, within the obvious limits of our assumptions $\left(0<c_{1}, c_{2}<c_{3}<c_{1}+c_{2}\right)$. Also, even with perfect negative correlation, the proposition shows that there are situations where one should not invest in flexible capacity (case (a) requires high flexible cost and low margin products) or where total capacity is less than total a priori known demand $k$ (case (b) allows 
The Optimal Investment Strategies When Demands Are Perfectly Negatively Correlated and $c_{1} / p_{1}+c_{2} / p_{2}>1$ for Three Scenarios: High Cost of Flexibility (Left), Medium Cost (Center), Low Cost (Right)
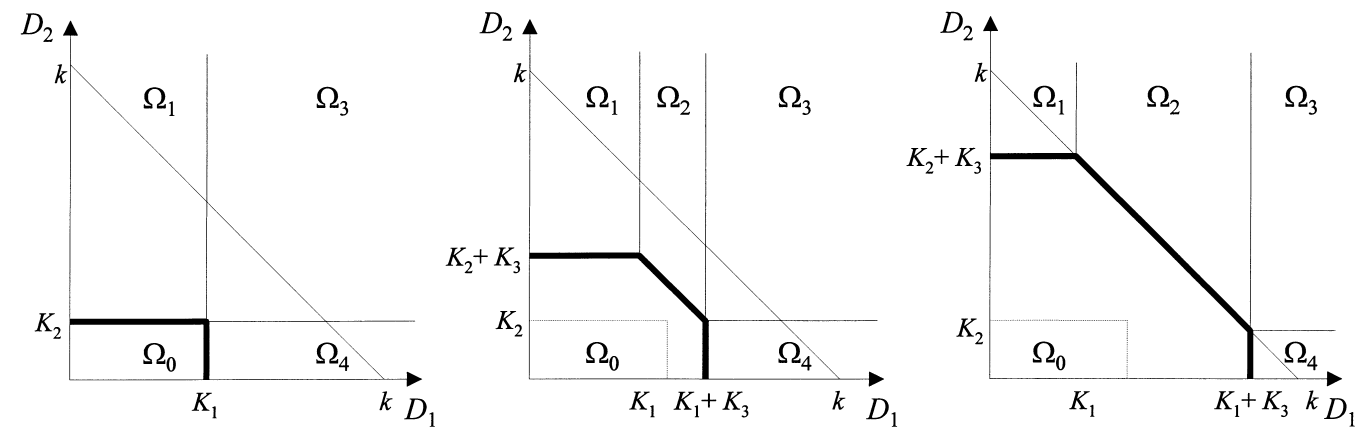

medium flexible cost if the price (margin) differential is high). These results, like those of Proposition 4, are strong in that the threshold values $\overline{c_{3}}$ and $c_{3}^{*}$ are independent of the probability distribution of $D_{1}=k-D_{2}$.

In Van Mieghem (1997) optimal investment plans are derived numerically for a particular family of demand distributions where two parameters, correlation and variability, can be varied continuously and independently. Extending the two boundary cases presented here, it appears that the optimal levels of dedicated capacity increase in a concave manner as correlation increases, while the optimal level of flexible capacity decreases in a convex manner. (The opposite usually, but not always, happens as variability increases.) This is the substitution effect implicit in Proposition 5. ${ }^{2}$

${ }^{2}$ I am grateful to Mike Harrison, Sunil Chopra and the anonymous referees for their helpful suggestions on preliminary versions.

\section{References}

Caulkins, J. P., C. H. Fine. 1990. Seasonal inventories and the use of product-flexible manufacturing technology. Annals of Operations Research 26 351-375.

Eberly, J. C., J. A. Van Mieghem. 1997. Multi-factor dynamic investment under uncertainty. J. of Economic Theory. 75 345-387.

Fine, C. H., R. M. Freund. 1990. Optimal investment in product-flexible manufacturing capacity. Management Science. 36 449-466.

Harrison, J. M., J. A. Van Mieghem. 1998. Multi-resource investment strategies: Operational hedging under demand uncertainty. $E u-$ ropean J. of Operational Research. 1-20. Forthcoming.

Jones, R. A., J. M. Ostroy. 1984. Flexibility and uncertainty. Review of Economic Studies. 51 13-32.

Li, S., D. Tirupati. 1994. Dynamic capacity expansion problem with multiple products: Technology selection and timing of capacity additions. Operations Research. 42 958-976.

Van Mieghem, J. A. 1997. Investment strategies for flexible resources. Technical report, Center for Mathematical Studies in Economics and Management Science, Northwestern University. Available at http:/ / www.kellogg.nwu.edu/research / math.

Accepted by Hau L. Lee; received May 4, 1995. This paper has been with the author 2.5 months for 2 revisions. 